\title{
Potential Drug-Drug Interactions among Prescribed Drugs in Paediatric Outpatients Department of a Tertiary Care Teaching Hospital
}

\author{
Malkesh Mistry, ${ }^{*}$ Alpa Gor, Barna Ganguly \\ Department of Pharmacology, Pramukhswami Medical College, Karamsad, Gujarat, INDIA.
}

\begin{abstract}
Objective: To evaluate prevalence, types and severity of drug-drug interactions in paediatric outpatient department. Methods: An observational study was carried out in Shree Krishna Hospital (SKH), a tertiary care teaching hospital after obtaining Institutional Ethics Committee approval. In SKH outpatient prescriptions are uploaded on software "Solace", and these online prescriptions were collected from IT department. Potential Drug-drug interactions (pDDIs) were identified by using Medscape drug interaction checker. The prescriptions were analysed for demographic characteristics, medical and detailed drug history. Drug-drug interactions (DDIs) were evaluated for total numbers, types and severity of DDIs. Result: Total 300 prescriptions with mean age $6.33 \pm 5.24$ years were analysed. Total 96 pDDIs were recorded. The prevalence of pDDI was $16 \%$. Most common pDDI was Valproic acid + Acetaminophen. There were 61 (63.54\%) DDIs of pharmacokinetic, 24 (25\%) pharmacodynamic and $11(11.46 \%)$ had an unknown mechanism. Out of all interactions, 41 (42.71\%) were of minor,
\end{abstract}

$48(50 \%)$ were of moderate and $07(7.29 \%)$ were of severe in nature. The number of drugs prescribed and the occurrence of DDIs were significantly associated. $(p<0.001)$

Key words: Drug-Drug Interactions, Paediatric, Pharmacokinetic, Pharmacodynamic, Prescription.

Correspondence :

Dr. Malkesh Harshadbhai Mistry, $3^{\text {td }}$ year resident, Department of Pharmacology, Pramukhswami Medical College, Karamsad-388325, Gujarat, INDIA.

Cell: +917600053438

Email:drmalkesh@gmail.com

DOI: 10.5530/jyp.2017.9.74

\section{INTRODUCTION}

Modern recent advances in different pharmacotherapies have helped doctors to improve quality of life of patients'. But with number of different groups of drugs available for certain conditions, selecting a proper drug has become a bigger problem. As a result, total number of drugs use are increasing. These drugs not always produce desirable effects, they can sometimes lead to unfavourable therapeutic effects. One of this unfavourable effect is potential drug-drug interactions. (DDIs). When a drug combination could lead to an unexpected change in the condition of the patient, this would be described as an interaction of potential clinical significance. ${ }^{1}$ Drug-drug interactions represent an important and widely under- recognized source of medication errors. DDIs responsible for $23 \%$ of hospital admission., ${ }^{2,3}$

Potential DDI is the qualitative or quantitative change in the effect of a drug either pharamacokinetically or pharmacodynamically. Pharmacodynamic interaction, involves receptor effects of different agents which interact to produce synergism or antagonism of drug effects. In pharmacokinetic interaction, the blood levels of given agents may be increased or decreased based on the potential to alter the pattern of absorption, distribution, metabolism and excretion. According to severity, drug interactions may be major interactions which may be life threatening. For moderate additional treatment, hospitalization, or extension of hospital stay may be necessary. And minor interactions, no additional treatment required.

Potential drug-drug interactions are those that can be predicted from the known pharmacological properties of the drugs involved. However, not all potential drug interactions necessarily have actual clinically significant consequences, particularly when drugs with wide therapeutic windows are involved. For example, many pharmacokinetic interactions, like drug displacement from plasma protein-binding sites, are often clinically unimportant. Also, potential drug-drug interactions do not necessarily occur in all patients, involving only subjects with specific characteristics, such as extensive or poor metabolizers of CYP isoenzyme. ${ }^{5}$

Majority of the drug-drug interaction studies involved adult patients. Studies revealed that paediatrics patients are at higher risk to druginduced problems because of many factors such as wide-ranging of patient ages and body-weights, limited physiologic reserve, calculation errors in medications dosing, and incapability to properly communicate with healthcare professionals. ${ }^{6}$ Therefore, there is an utmost need to gather data regarding drug-drug interactions in paediatric population

The study initiated with an aim to evaluate prevalence, types of drugdrug interaction and severity of drug-drug interactions in paediatric outpatient department of a tertiary care teaching hospital.

\section{MATERIALS AND METHODS}

A cross sectional observational study was carried out in paediatric outpatient department of Shree Krishna Hospital (SKH), a tertiary care teaching hospital after obtaining ethical approval from institutional ethics committee. Study began after getting permission from head of paediatric department to collect online prescriptions. In SKH outpatient prescriptions are uploaded on software "Solace", and these online prescriptions were collected from IT (information technology) department for the study. All the patients visiting at paediatric outpatient department of a tertiary care teaching hospital was included in the study. The patients

This is an open access article distributed under the terms of the Creative Commons Attribution-NonCommercial-ShareAlike 4.0 License, which allows others to remix, tweak, and build upon the work non-commercially, as long as the author is credited and the new creations are licensed under the identical terms. 
whose prescription contain only one drug were excluded. (Food-drug interaction and herbal drug interactions are not checked). Confidentiality about patients' identification was maintained.

Total 300 online prescription were collected. All the prescriptions were selected by simple randomization. Demographic data (age, gender, and body weight), medical history, co-morbidities and drug related data (name of drug, dose, duration, frequency, route and concomitant medication) were recorded in a specially designed proforma.

All collected prescriptions were evaluated for DDIs by the online Medscape drug interaction checker. ${ }^{7}$ DDI were also checked by referring standard text books of pharmacology (Goodman and Gilman's The Pharmacological Basis of Therapeutics, $12^{\text {th }}$ edition). The prescriptions were analysed for different variables of DDIs like total numbers of interactions, types and severity. All the significant DDIs were classified based on mechanism like pharmacodynamics, pharmacokinetic and unknown. The severity of DDIs was classified as minor, moderate and serious.

\section{Statistical Analysis}

Descriptive statistics, mean, standard deviation was applied for analysing the data. The Statistical software namely SPSS 20.0 and Stata 14 were used for the analysis of the data and Microsoft word and Excel had been used to generate graphs, tables etc.

\section{RESULTS}

The study population was classified in five different age groups, 0 - 1 month (1.33\%), 2 months - 1year (24.67\%), 2-6 years (31.33\%), 7-12 years $(24.33 \%)$ and $13-18$ years $(18.33 \%)$. Mean age of patient was $6.33 \pm 5.24$ years. Among them total 133 (44.33\%) were females and 167 (55.67\%) were males.

All 300 prescriptions had one morbidity per prescription. The most common morbidity was gastrointestinal and chemotherapy $(n=63)$ followed by respiratory $(n=59)$, genitourinary $(n=25)$.

Among all the prescriptions, total 857 number of drugs were prescribed. Maximum prescriptions $44.33 \%(n=133)$ had two drugs per prescription followed by $37 \%(n=111)$ had three drugs per prescription and $12.67 \%$ $(n=38)$ had four drugs per prescription. Maximum eight drugs were prescribed in only one prescription.

Total 96 DDIs were observed. An average of $2.85 \pm 1.04$ drugs was prescribed per prescription with an average of $0.32 \pm 0.93$ potential DDIs were observed. No direct correlation was observed between the age of the patient and the number of drugs prescribed, the age of the patient and the number of DDIs. However, the number of drugs prescribed and the occurrence of DDIs were significantly associated $(r=0.3458$, $\mathrm{p}<0.001)$. A positive correlation was observed as the number of DDIs increases as the number of prescribed drug increases.

Out of 300 prescriptions, 252 (84\%) prescriptions had no drug-drug interactions. Maximum number of prescriptions, $26(8.67 \%)$ had one drug-drug interaction, followed by $10(3.33 \%)$ prescriptions had three interactions and 06 (2\%) prescriptions had four interactions. Most number of DDIs in a single prescription was six, which were recorded in three prescriptions.

DDIs were classified based on mechanism like pharmacodynamics, pharmacokinetic and unknown. [Figure 1]

Maximum interactions 48 (50\%) were of moderate severity followed by 41 (42.71\%) were of minor severity and 07 (7.29\%) were of severe severity. Most frequently involved serious DDI was Rifampin + Pyrazinamide $(n=3)$ followed by Rifampin + Isoniazid $(n=2)$, Ferrous Fumarate + Levofloxacin $(n=1)$ and Fentanyl + Propofol $(n=1)$. Other most commonly involved moderate DDIs were Chlorpheniramine + Phenylephrine $(n=6)$, Ferrous fumarate + Ciprofloxacin $(n=3)$ and Ferrous sulphate +

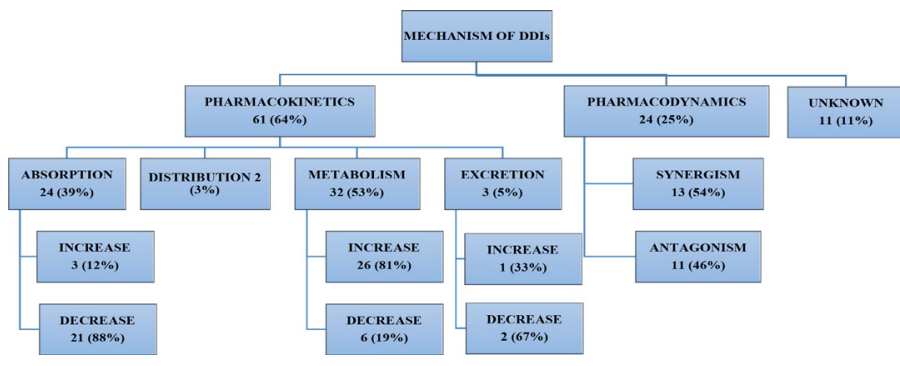

Figure 1: DDIs based on their mechanism

Leothyroxine $(\mathrm{n}=3)$. And most commonly involved minor DDIs were Valproate + Acetaminophen $(n=6)$, Valproate + Phenobarbital $(n=5)$ and Oxcarbazapine + Acetaminophen $(\mathrm{n}=4)$.

\section{Pharmacokinetic drug-drug interactions}

Total 24 interactions could affect absorption, out of which 03 (12.5\%) drug and $21(87.5 \%)$ drug could decrease could increase the level or effect of other drug by increasing or decreasing gastric absorption. Of all 61 pharmacokinetic interactions observed, 02 (3.28\%) interactions could affect distribution. Total 32 interactions could affect metabolism, out of which $26(81.25 \%)$ drug combinations could decrease the level of other drug and $06(18.75 \%)$ could increase the level of another drug by increasing or decreasing metabolism. Of the 32 DDIs, which could affect the metabolism of drug, 08 were shown to affect CYP450 enzyme subtypes. Most common CYP450 enzyme involved in these interactions were CYP2C9 [Valproic acid + Phenytoin ( $\mathrm{n}=2)$, Phenobarbital + Phenytoin $(n=2)$, Metronidazole + Ibuprofen $(n=1)$ ] followed by CYP2C19 [Oxcarbazepine + Escitalopram $(\mathrm{n}=1)$, Rifampin + Pantoprazole $(\mathrm{n}=1)]$ and CYP2E1 [Isoniazid + Acetaminophen $(n=1)$ ]. Total 03 interactions could affect excretion of the drug, out of which $02(66.67 \%)$ drug increase and $01(33.33 \%)$ drug decreases levels of other drug by increasing or decreasing renal clearance. [Table 1]

\section{Pharmacodynamics drug-drug interactions}

Out of all 24 pharmacodynamic interactions, 13 showed pharmacodynamic synergism i.e. increase serum calcium level in two prescriptions, to increase risk of hepatotoxicity in five prescriptions, increase toxicity of one drug in one prescription, prolong QTc interval in one prescription, increase sedative effect by both drugs in two prescriptions, decrease sedative effect by both drugs in two prescriptions. [Table 2]

A total of 11 interactions showed pharmacodynamic antagonism. Among them one interaction showed an alteration of serum potassium level, ten interactions showed an alteration in sedative effect. [Table 3] Of all 96 DDIs, Chlorpheniramine + Phenylephrine $(n=6)$ and Valproic Acid + Acetaminophen $(n=6)$ were most commonly observed interactions.

\section{DISCUSSION}

Drug - drug interactions are one of the main causes of adverse reactions related to medications, which is responsible for $23 \%$ of hospital admission and also associated with increased health care costs. ${ }^{2,3,4-8,9}$ Data regarding to prevalence, type and severity of DDIs in paediatric age group in Indian set up is limited. The present study was conducted with aim and objective to identify and evaluate the drug-drug interactions in paediatric outpatient department.

In present study prevalence of DDIs were more in male as compare to females which was contrast to the study done by Feinstein $\mathrm{J}$ et al ${ }^{10}$ showed equal occurrence of DDIs in male and female. It suggests that their prevalence could differ in individual gender. 
Table 1: Drug combinations showing pharmacokinetic drug-drug interactions

\begin{tabular}{lc}
\hline \multicolumn{1}{c}{ Pharmacokinetic drug interactions } \\
\hline \multicolumn{1}{c}{ Drug combinations which increase absorption } & Frequency \\
\hline Metoclopramide + Acetaminophen & 1 \\
Ferrous Sulfate + Calcium Carbonate & 1 \\
Ferrous Fumarate + Calcium Carbonate & 1 \\
Drug combinations which decrease absorption & \\
Ferrous Fumarate + Ciprofloxacin & 3 \\
Calcium Carbonate + Ferrous Fumarate & 3 \\
Pantoprazole + Ferrous Fumarate & 2 \\
Others & 13 \\
Drug combinations affecting distribution & \\
Valproic Acid + Phenytoin & 2 \\
Drug combinations which increase metabolism & \\
Valproic Acid + Acetaminophen & 6 \\
Oxcarbazepine + Acetaminophen & 4 \\
Carbamazepine + Acetaminophen & 4 \\
Others & 12 \\
Drug combinations which decrease metabolism & \\
Valproic Acid + Phenytoin & 1 \\
Isoniazid + Acetaminophen & 2 \\
Metronidazole + Ibuprofen & 1 \\
Others & \\
Drug combinations which increase excretion & \\
Prednisolone + Calcium Carbonate & \\
Drug combinations which decrease excretion & \\
Cefadroxil + Ibuprofen & \\
Aluminum Hydroxide + Magnesium Trisalicylate & \\
\hline & \\
\hline
\end{tabular}

Table 2: Drug combinations showing synergistic pharmacodynamic drug-drug interactions

\begin{tabular}{lc}
\hline Pharmacodynamics DDIs on effect of electrolyte balance & \\
\hline Hypercalcemia & Frequency \\
\hline Vitamin D + Calcium Carbonate & 2 \\
Pharmacodynamics DDIs with increased risk of toxicity & \\
Increased risk of hepatotoxicity & 3 \\
Rifampin + Pyrazinamide & 2 \\
Isoniazid + Pyrazinamide & \\
Increase toxicity of other drug & 1 \\
Fentanyl + Propofol & \\
Prolongation of QTc interval & 1 \\
Hydroxyzine + Azithromycin & \\
Increase sedative effect & \\
Chlorpheniramine + Hydroxyzine & 1 \\
Propofol + Midazolam & 1 \\
Decrease sedative effect & \\
Caffeine + Phenylephrine &
\end{tabular}

Table 3: Drug combinations showing antagonistic pharmacodynamic drug-drug interactions

\begin{tabular}{lc}
\hline Fluctuation in serum potassium level & Frequency \\
\hline Potassium Chloride + Levalbuterol & 1 \\
Alter sedative effect & \\
Chlorpheniramine + Phenylephrine & 6 \\
Chlorpheniramine + Caffeine & 3 \\
chlorpheniramine + levalbuterol & 1 \\
\hline
\end{tabular}

A retrospective study by Lin et al. ${ }^{11}$ in outpatients in Taiwan emphasis that polypharmacy increases the risk of DDIs. In present study, maximum number of patients prescribed two drugs per prescription. Whereas, study done in Pakistan, ${ }^{12}$ maximum number of patients had prescribed 4-6 drugs per prescription. The potential risk of DDIs increases with an increase number of drugs prescribed. ${ }^{14}$ Thus polypharmacy should be avoided to minimise the risk of DDIs.

In present study, the overall prevalence of DDIs was $16 \%$ out of all DDIs. Prevalence rate was lower in compare to studies conducted by Feinstein et $a l^{10}$ and Qorraj-Bytyqi et al., ${ }^{12}$ they reported that paediatric patients exposed to DDI were $49 \%$ and $23 \%$ respectively.

DDI severity criteria enhances decision making ability by assessing risk versus benefit alternatives. Minor drug interactions do not result in any troublesome outcomes and management usually not required. Moderate drug interactions could result worsening in clinical condition of patient. Treatment to manage such type of interactions could be considered. Major drug interactions could lead to life threatening condition; therefore it should be considered essential to counter such problems as soon as they are identified. ${ }^{15}$ In present study maximum interactions were moderate in nature followed by mild and serious interactions. The study conducted by Oshikoya $\mathrm{KA}^{16}$ found most DDIs were major and serious followed by moderate and clinically significant, which was different to the present study. In these cases, vigorous monitoring of prescriptions and awareness of interactions is needed to prevent the side effects which indirectly reduce the cost of the therapy.

In present study, most of the interactions were pharmacokinetic, followed by pharmacodynamic interactions. There were few interactions which were having unknown mechanism, which was similar to the study conducted by Get chew $\mathrm{H}$ et al. ${ }^{18}$

Drug interactions can alter the absorptions in terms of either increased or decreased absorption, altered gastric emptying and altered gut flora. Altered absorption due to DDIs can reduce the concentrations of another drug. The most common interactions which leads to increase in absorption of the other drug were detected with prokinetics, iron salts, calcium supplements and non-steroidal anti-inflammatory drugs (NSAIDs). As these are the most frequently prescribing drugs, careful selection of drugs is always necessary to reduce the risk of toxicity. The decrease level or effect of the other drug by decreasing gastric absorption, which was commonly detected with $\mathrm{H}_{2}$ blockers, fluoroquinolones, macrolides, and proton pump inhibitors (PPIs) and iron salts. The rate and extent of absorption may be important, in the case of drugs given in single doses where a threshold concentration for drug effect exists (e.g. analgesics, antibiotics).${ }^{13}$ A delay in absorption in these circumstances, especially if the rate of elimination of the drug is high, may result in failure of therapeutic efficacy or drug resistance. ${ }^{13}$

In drug displacement interaction, there is a reduction in extent of plasma protein binding of one drug by the presence of another which competes for the same binding sites. In this study, potential alteration of distribution of one drug by another drug by plasma protein binding competition 
was most common between Anti-epileptics. These drugs have high plasma protein binding, and drug displacement reactions are more common with drugs having high plasma protein binding. Such drug displacement can result in toxicity and the effects of such interactions may need to be taken into account in therapeutic drug monitoring.

The clinical significance of increased drug metabolism is decreased plasma level of the co-administered drug. Similarly decrease metabolism of other drug resultant increase in level of slowly metabolised drug and prolongation of its effect. ${ }^{19}$ In both the cases alteration in dosing and close monitoring is required to avoid unnecessary ADRs. A potential chance of increased metabolism was observed between groups of drugs like anti-epileptics, anti-tubercular drugs and NSAIDs. And some of the group of drugs were observed to decrease metabolism of other drugs like anti-epileptics, NSAIDs and selective serotonin reuptake inhibitors (SSRIs).

DDIs can lead to change in metabolism of other drugs, own metabolism or particular drug by either enzyme induction or inhibition. The Cytochrome P 450 (CYP450) enzymes plays an important role in the biotransformation of a wide number of drugs. Many drugs that undergo CYP mediated oxidative biotransformation is responsible for the large number of clinically significant DDIs during multiple drug therapy. ${ }^{20 \text {, }}$ ${ }^{21}$ In present study the most common CYP450 enzyme involved in the interactions were CYP2C9 followed by CYP2C19 and CYP2E1. Genetic polymorphism of CYP plays an important role in therapeutic effect of drug treatment. ${ }^{22,23}$ It signifies that the therapeutic drug monitoring in patients with multiple drug treatment and in vitro techniques can predict the role of CYP enzymes polymorphism, and decrease the occurrence of DDIs. ${ }^{21}$

Majority of the interactions involving elimination or excretion process occur due to change in urinary $\mathrm{pH}$, changes in active renal tubule excretion and different drug transporter protein. ${ }^{13}$ Increase in excretion of one drug by another drug was detected between steroids and calcium supplements. Similarly decrease in excretion of another drug was detected between groups of drugs as cephalosporins with NSAIDs and with different antacids. DDIs due to altered excretion process, can lead to toxicity or sub therapeutic effect.

The pharmacodynamic interaction of a drug can be altered by competition at receptors, and non-receptor. Pharmacodynamics interactions can occur when two drugs have similar actions through different cellular mechanisms. ${ }^{17}$ In present study almost $25 \%$ of interactions from total, observed due to pharmacodynamic changes either by synergism or antagonism.

Evidence of electrolyte disturbance like hypercalcemia was also observed in present study by group of drugs like vitamin $\mathrm{D}$ and calcium salts. Ethiopian study by Admassie et $a^{24}$ also observed the similar results. The concurrent use of vitamin D with calcium salts is generally beneficial, in some patients this combination may result in hypercalcemia. ${ }^{25}$

Another risk of toxicity like hepatotoxicity was observed by anti-tubercular drugs. A meta-analysis also suggest that combination of anti-tubercular drugs are associated with higher rate of hepatotoxicity. ${ }^{26}$ Possible mechanism is due to rifampin enhances the metabolism of isoniazid to hepatotoxic metabolites. In such cases, close monitoring of each individual and dose adjustment is recommended.

There was a pharmacodynamics interactions leading to increase in QTc interval. In present study the drugs causing prolongation of QTc interval was macrolide and anti-histaminic. These interactions led to the restriction or withdrawal of many drugs in different countries. The underlying mechanism is thought to involve drug-induced blockade of the repolarizing potassium channels, particularly the inward rectifier, causing prolongation of the action potential and duration and early after- depolarization. ${ }^{13}$ Prolongation of the QTc interval can cause torsades de pointes arrhythmia, which can lead to ventricular fibrillation and sudden death. To avoid such serious interactions these types of combinations should be avoided or replaced with another drug.

One of the beneficial antagonistic effect observed was altered in serum potassium level by the drugs. The commonest group of drugs involved was $\beta_{2}$ adrenergic receptor agonist and potassium chloride. Kapadia J et $\mathrm{al}^{27}$ had observed the same pattern of beneficial antagonist effect.

The strength of the study was, to generate local data regarding potential DDIs. The study provides the baseline data for future studies of in inpatient or outpatient in different departments or in different institute. These particular results regarding occurrence of potential DDIs will be notified to the physicians to sensitize them.

\section{LIMITATION}

In present study, the utilization of DDI-checker software provides only a 'potential' estimate of DDI occurrence. Further follow up studies can be planned to identify ADRs due DDIs. Present study was done by using a freely available DDI checker on Internet, but it can be planned by the use of more than one drug interaction checker to produce more precise results. However, despite these limitations, the study approach is currently widely used to assess the clinical relevance and risk of exposure to potential DDIs.

\section{CONCLUSION}

Potential DDIs increased with an increased number of drug prescribed. The use of electronic interaction software, continuing education and observation by prescribers towards drug selection may decrease the problem of potential adverse DDIs. The use of most commonly involved drug groups in adverse DDIs should be reduced or change with the alternative drugs.

\section{ACKNOWLEDGEMENT}

Authors are highly thankful to IT department for retrieval of data and Mr. Mayur Shinde for helping in analysis of data.

\section{CONFLICT OF INTEREST}

Authors do not have any conflict of interest.

\section{ABBREVIATION USED}

DDIs: Drug-drug interaction; CYP450: Cytochrome P 450; NSAIDs: Non-steroidal anti-inflammatory drugs; PPIs: Proton pump inhibitors; ADRs: Adverse drug reactions; SSRIs: Selective serotonin reuptake inhibitors.

\section{REFERENCES}

1. Bista D, Palaian S, Shankar PR, Prabhu MM, Paudel R, Mishra P. Understanding the essentials of drug interactions: a potential need for safe and effective use of drugs. Kathmandu Univ Med J (KUMJ). 2007;5(3):421-30.

2. Adverse Drug Reactions and Drug-Drug Interactions: Consequences and Costs | AMFS [Internet]. Amfs.com. 2016 [cited 8 November 2016]. Available from: http://www.amfs.com/news/articles-from-our-experts/adverse-drug-reactionsand-drug-drug-interactions-consequences-and-costs/.

3. Helms RA, Quan DJ, editors. Textbook of therapeutics: drug and disease management. Lippincott Williams \& Wilkins; 2006

4. Lubinga SJ, Uwiduhaye E. Potential drug-drug interactions on in-patient medication prescriptions at Mbarara Regional Referral Hospital (MRRH) in western Uganda: prevalence, clinical importance and associated factors. Afr Health Sci. 2011;11(3). PMid:22275946 PMCid:PMC3261003.

5. Magro L, Moretti U, Leone R. Epidemiology and characteristics of adverse drug reactions caused by drug-drug interactions. Expert Opin Drug Saf 2012;11(1):83-94. https://doi.org/10.1517/14740338.2012.631910;PMid:22022824.

6. Wang JK, Herzog NS, Kaushal R, Park C, Mochizuki C, Weingarten SR. Preven- 
tion of pediatric medication errors by hospital pharmacists and the potential benefit of computerized physician order entry. Pediatrics. 2007;119(1):e77-85. https://doi.org/10.1542/peds.2006-0034; PMid:17200262.

7. Multi-Drug Interaction Checker [Internet]. Reference.medscape.com. 2016 [cited 01 January 2017]. Available from: http://reference.medscape.com/druginteractionchecker

8. Hamilton RA, Briceland LL, Andritz MH. Frequency of Hospitalization after Exposure to Known Drug-Drug Interactions in a Medicaid Population. Pharmacotherapy: J Human Pharmacol Drug Ther. 1998;18(5):1112-20.

9. Jankel CA, McMillan JA, Martin BC. Effect of drug interactions on outcomes of patients receiving warfarin or theophylline. Am J Health Syst Pharm. 1994;51(5):661-6.

10. Feinstein J, Dai D, Zhong W, Freedman J, Feudtner C. Potential drug-drug interactions in infant, child, and adolescent patients in children's hospitals. Pediatrics. 2015;135(1):e99-108.

11. Lin $\mathrm{CF}$, Wang $\mathrm{CY}$, Bai $\mathrm{CH}$. Polypharmacy, aging and potential drug-drug interactions in outpatients in Taiwan. Drugs \& aging. 2011;28(3):219-25. https://doi. org/10.2165/11586870-000000000-00000; PMid:21250763.

12. Qorraj-Bytyqi H, Hoxha R, Krasniqi S, Bahtiri E, Kransiqi V. The incidence and clinical relevance of drug interactions in pediatrics. J Pharmacol Pharmacother [serial online] 2012 [cited 2017 Jan 31];3:304-7. Available from: http://www. jpharmacol.com/text.asp?2012/3/4/304/103686.

13. Van Boxtel C, Santoso B, Edwards I. Drugs benefit and risk. $2^{\text {nd }}$ ed. Amsterdam: IOS Press; 2008.

14. Tripathi KD. Essentials of medical pharmacology. JP Medical Ltd; 2013 Sep 30.

15. Ahmad A, Khan MU, Haque I, Ivan R, Dasari R, et al. Evaluation of potential drug-drug interactions in general medicine ward of teaching hospital in southern India. J Cli Diagn Res. 2015;9(2). https://doi.org/10.7860/jcdr/2015/11264.5608.

16. Oshikoya KA, Oreagba IA, Godman B, Oguntayo FS, Fadare J, et al. Potential drug-drug interactions in paediatric outpatient prescriptions in Nigeria and implications for the future. Expert Rev of Clin Pharmacol. 2016;9(11):1505-15. https:// doi.org/10.1080/17512433.2016.1232619; PMid:27592636.

17. Brunton LL, Chabner BA, Knollmann BC. Goodman \& Gilman's The Pharmacological Basis of Therapeutics. 12 $2^{\text {th }}$ ed2011.
18. Getachew H, Assen M, Dula F, Bhagavathula AS. Potential drug-drug interactions in pediatric wards of Gondar University Hospital, Ethiopia: A cross sectional study. Asian Pac J of Trop Biomed. 2016;6(6):534-8.https://doi.org/10.1016/j. apjtb.2016.04.002.

19. Sharma KK and Sharma HL. Principle of Pharmacology. $2^{\text {nd }}$ edition. Paras medical publisher 2011.

20. Srivastava S. A complete textbook of Medical Pharmacology. $1^{\text {st }}$ ed. Kala Amb: Avichal Publishing Company; 2012.

21. Palleria C, Di Paolo A, Giofrè C, Caglioti C, Leuzzi G, et al. Pharmacokinetic drug-drug interaction and their implication in clinical management. J Res Med Sci. 2013;18(7):601.

22. Gallelli L, Colosimo M, Tolotta GA, Falcone D, Luberto L, Curto LS, et al. Prospective randomized double-blind trial of racecadotril compared with loperamide in elderly people with gastroenteritis living in nursing homes. Eur $\mathrm{J}$ Clin Pharmacol. 2010;66(2):137-44. https://doi.org/10.1007/s00228-009-0751-3; PMid:19902197.

23. Gallelli L, Galasso O, Urzino A, Saccà S, Falcone D, Palleria C, et al. Characteristics and clinical implications of the pharmacokinetic profile of ibuprofen in patients with knee osteoarthritis. Clin Drug Inv. 2012;32(12):827-33. https://doi. org/10.1007/s40261-012-0011-7; PMid:23086696.

24. Admassie E, Melese T, Mequanent W, Hailu W, Srikanth BA. Extent of polypharmacy, occurrence and associated factors of drug-drug interaction and potential adverse drug reactions in Gondar Teaching Referral Hospital, North West Ethiopia. J Adv Pharm Tec Res. 2013;4(4):183.

25. Sunyecz JA. The use of calcium and vitamin $D$ in the management of osteoporosis. Ther Clin Risk Manag. 2008;4(4):827-36.

26. Ramappa V, Aithal GP. Hepatotoxicity related to anti-tuberculosis drugs: mechanisms and management. J Clin Exp Hepatol. 2013;3(1):37-49. https://doi. org/10.1016/j.jceh.2012.12.001; PMid:25755470 PMCid:PMC3940184.

27. Kapadia J, Thakor D, Desai C, Dikshit RK. A study of potential drug-drug interactions in indoor patients of medicine department at a tertiary care hospital. J Applied Pharmaceutical Sci. 2013;3(10):89.

Article History: Submission Date : 12-01-2017; Revised Date : 07-03-2017; Acceptance Date : 04-05-2017.

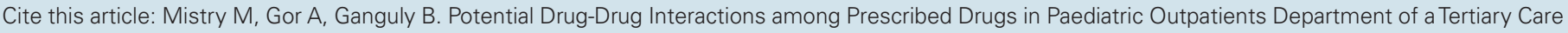
Teaching Hospital. J Young Pharm. 2017;9(3):371-5. 